\title{
A systematic review of physician retirement planning
}

Michelle Pannor Silver ${ }^{1,2^{*}}$, Angela D. Hamilton ${ }^{1}$, Aviroop Biswas ${ }^{2}$ and Natalie Irene Warrick ${ }^{2}$

\begin{abstract}
Background: Physician retirement planning and timing have important implications for patients, hospitals, and healthcare systems. Unplanned early or late physician retirement can have dire consequences in terms of both patient safety and human resource allocations. This systematic review examined existing evidence on the timing and process of retirement of physicians. Four questions were addressed: (1) When do physicians retire? (2) Why do some physicians retire early? (3) Why do some physicians delay their retirement? (4) What strategies facilitate physician retention and/or retirement planning?

Methods: English-language studies were searched in electronic databases MEDLINE, Web of Science, Scopus, CINAHL, AgeLine, Embase, HealthSTAR, ASSA, and PsycINFO, from inception up to and including March 2016. Included studies were peer-reviewed primary journal articles with quantitative and/or qualitative analyses of physicians' plans for, and opinions about, retirement. Three reviewers independently assessed each study for methodological quality using the Newcastle-Ottawa Scale for quantitative studies and Critical Appraisal Tool for qualitative studies, and a fourth reviewer resolved inconsistencies.

Results: In all, 65 studies were included and analyzed, of which the majority were cross-sectional in design. Qualitative studies were found to be methodologically strong, with credible results deemed relevant to practice. The majority of quantitative studies had adequate sample representativeness, had justified and satisfactory sample size, used appropriate statistical tests, and collected primary data by self-reported survey methods.

Physicians commonly reported retiring between 60 and 69 years of age. Excessive workload and burnout were frequently cited reasons for early retirement. Ongoing financial obligations delayed retirement, while strategies to mitigate career dissatisfaction, workplace frustration, and workload pressure supported continuing practice.

Conclusions: Knowledge of when physicians plan to retire and how they can transition out of practice has been shown to aid succession planning. Healthcare organizations might consider promoting retirement mentorship programs, resource toolkits, education sessions, and guidance around financial planning for physicians throughout their careers, as well as creating post-retirement opportunities that maintain institutional ties through teaching, mentoring, and peer support.
\end{abstract}

\section{Background}

Over the last 40 years, across multiple jurisdictions, a pattern has emerged whereby a disproportionate number of physicians continue to practice beyond the traditional retirement age of approximately 65 years old $[1,2]$. Accordingly, healthcare organizations often do not have effective succession strategies in place to manage their

\footnotetext{
* Correspondence: michelle.silver@utoronto.ca

${ }^{1}$ Department of Anthropology/Health Studies, University of Toronto Scarborough Campus, 1265 Military Trail, Toronto, Ontario M1C 1A4, Canada

${ }^{2}$ Institute of Health Policy, Management and Evaluation, University of

Toronto, Toronto, Ontario, Canada
}

aging medical staff. The consequences of an older physician workforce can be dire and far-reaching. Replacing invaluable and experienced older physicians with trained but inexperienced younger physicians can be difficult [3]. In addition, the link between advancing age and deteriorating health may lead to increased medical errors, putting patient health at risk [4].

For an experienced physician, the decision regarding when to transition from practice to retirement can be about more than clinical [5] and technological competency [6], it can also involve internal emotional struggles. This is particularly the case when individuals have a 
strong sense of value attached to their work [7]. Evidence suggests that physicians' adjustment to later career transitions can be facilitated by planning for retirement [8]. The objective of this review was to examine when physicians retire, why they retire early or delay retirement, and what strategies exist to facilitate physician retention and retirement planning. To our knowledge, no earlier studies have consolidated the literature with these questions in mind amidst the widespread call in the literature for such recommendations [9].

\section{Methods}

The Preferred Reporting Items for Systematic Reviews and Meta-Analyses (PRISMA) guidelines were followed in the production and reporting of this systematic review [10].

\section{Study selection}

Published articles were comprehensively searched using MEDLINE, Web of Science, Scopus, CINAHL, AgeLine, Embase, HealthSTAR, ASSA, and PsycINFO databases from inception up to and including March 2016. Our search strategy included the keywords "physician" and "retire" with all appropriate synonyms. All authors participated in the identification and final selection of studies.

\section{Study eligibility}

The PRISMA flow diagram in Fig. 1 depicts the numbers of identified records, excluded articles, and included studies. Our inclusion criteria included published primary peer-reviewed journal articles with quantitative and/or qualitative analyses of physicians' plans for, and opinions about, retirement. Excluded studies were non-primary research studies (editorials and commentaries), articles that grouped physicians with other healthcare professionals, or that only included dentists. After discussion, all authors agreed to constrain the search strategy to English-language articles, with no limitations on publication date up to March 2016. The search was supplemented by handsearching the references of eligible studies and relevant review articles.

\section{Data extraction}

The following information was extracted from qualifying studies: (i) geographic information, study design, data collection methodology, response rate, physician specialty; (ii) expected and actual retirement age; (iii) descriptive statistics related to demographic characteristics of the sample; and (iv) findings related to reasons for retiring, reasons for delaying retirement and obstacles to continued practice.

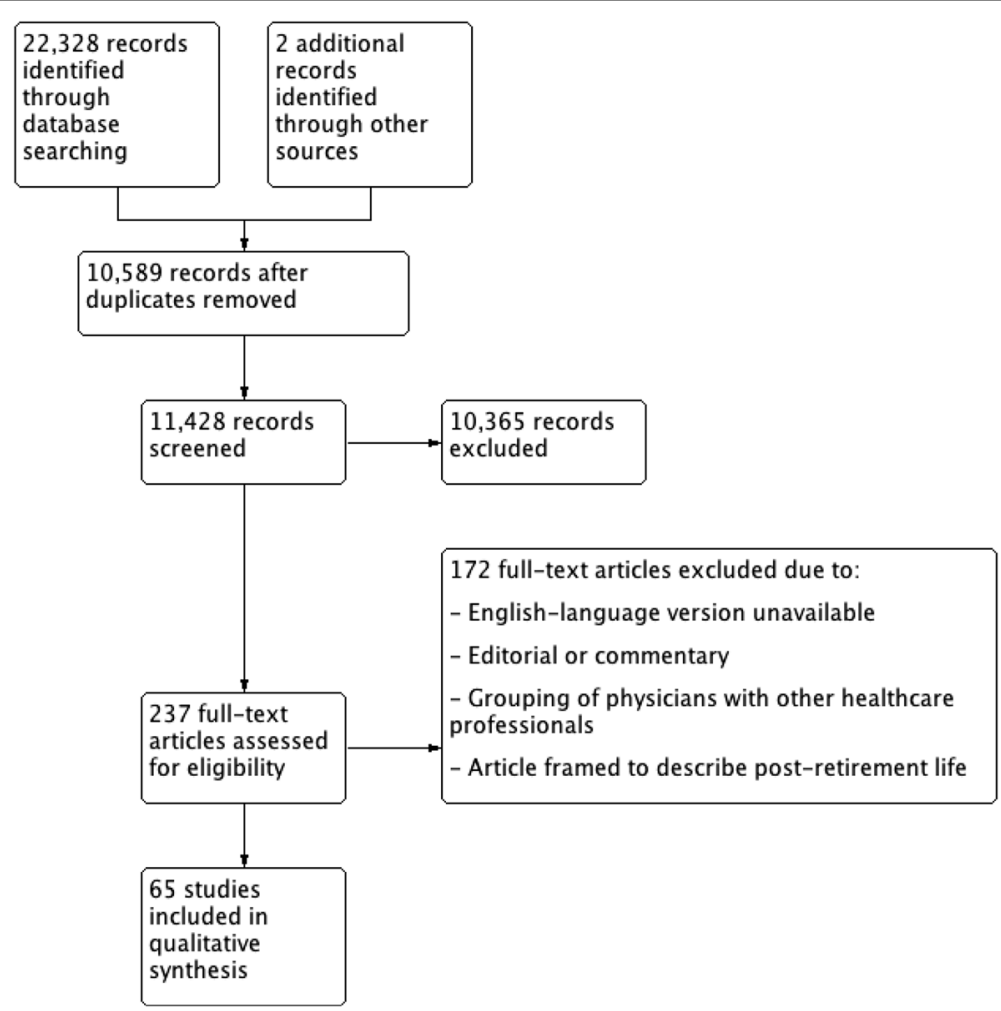

Fig. 1 PRISMA flow diagram 


\section{Quality assessment}

Three authors (ADH, $\mathrm{AB}$, and NW) worked in pairwise rotation to independently review qualifying articles for methodological quality. The corresponding author (MPS) resolved any disagreements that could not be settled by consensus. We used the seven-item, NewcastleOttawa Quality Assessment Scale to assess the risk of bias for the 55 studies that had used quantitative methods [11]. The adapted Critical Appraisal of a Qualitative Study Tool from the Center for Evidence-Based Management was used to assess 10 studies that used qualitative methods [12]. All studies examined by quality assessment were given equal weighting and both quality assessment tools were selected on the basis of previously demonstrated reliability and validity when examining the views of healthcare professionals [13-15].

\section{Terminology}

Early retirement is referred to in this study as either retirement that occurs earlier than the physician had planned [16] or to an exit from their profession at a relatively early age (i.e., younger than age 65 ) as compared to peers [17]. On time retirement refers to the conventional age of retirement, that is, at or around age 65 [18]. The literature commonly refers to retirement as late or delayed if physicians continue to work in a full-time capacity beyond the traditional age of retirement [19].

\section{Synthesis}

Thematic analysis was used to identify and stratify concepts related to physician retirement timing into themes and subthemes [20]. Thematic analysis is an inductive qualitative data analysis process in which data are prepared, then organized using open coding to create categories and themes to build a conceptual understanding of a particular phenomenon and analyze the meaning of data within their particular context [21].

\section{Results}

\section{Study characteristics}

Table 1 summarizes the characteristics of the 65 studies included in this review. The studies were published between 1978 and 2015, with 33 studies based in the United States, others in Australia, Canada, Finland, Israel, Netherlands, New Zealand, the United Kingdom, and one across 20 countries of high-, medium-, and lowincome economies. A variety of practicing and retired physicians were sampled with a range of specializations from general and multidisciplinary physicians to anesthesiologists, dentists, general and specialist surgeons, obstetrician-gynecologists, otolaryngologists, ophthalmologists, pediatricians, psychologists, radiologists, and urologists.
Tables 2 and 3 summarize the quality of the included studies. Qualitative studies [9, 18, 22-29] were found to be methodologically strong, with credible results deemed relevant to practice. The majority of quantitative studies had adequate sample representativeness (76\% of studies), had justified and satisfactory sample size ( $89 \%$ of studies), used appropriate statistical tests (59\% of studies), and collected primary data by self-reported survey methods ( $91 \%$ of studies). Studies were rated poorly on the ascertainment of exposure (i.e., how the outcome of interest was obtained either by secure record, structured interview, or self-reported) due to the use of nonvalidated measurement tools (51\% of studies). Nearly half $(49 \%)$ of the studies were rated poorly for comparability since they did not control for any potential confounders.

\section{Physician retirement age}

Physicians' actual retirement age and their intended or planned retirement age are distinguished in Table 4. Physicians' intended or planned retirement age refers to the age they speculate they will most likely be when they reach retirement [30]. This differs from physicians' actual retirement age, represented by the chronological age at which they reported being fully retired [31]. Comparisons of on-time, early, and delayed retirement were made in a context relative to physician peers [16, 32, 33] and across subspecialties [31, 34]. In some instances, comparisons were made to other professional groups such as social workers [35].

Our findings suggest the average age for actual and expected retirement was commonly reported to be between 60 and 69 years, respectively. Several studies $[7,18,22,30,31,36-39]$ examined the age that physicians expected to retire, and the age they actually retired (underlined in Table 4). The actual retirement age was found to be consistent with their expected retirement in all studies where the actual and expected retirement ages were jointly reported. These studies highlight that a variety of methods are used to determine usual age at retirement and that physicians' retirement intentions can, but not always, translate into actual retirement behaviors.

\section{Reasons for retiring early and obstacles to practice}

Common reasons for retiring early included low job satisfaction, medicolegal issues, health concerns, and financial troubles. Low job satisfaction involved perceptions of low job control, low morale, and dissatisfaction with the internal justice system of medicine as a self-regulated profession $[5,9,28,40]$. This disillusionment was expressed by a sense of frustration with colleagues [27, 35], feeling undervalued, lacking prestige [16, 41], and a loss of interest in their work [10]. Excessive workload [17, 42] and burnout 
Table 1 Characteristics of included studies

\begin{tabular}{|c|c|c|c|c|}
\hline Study & Location & $\begin{array}{l}\text { Study method (source, if not self- } \\
\text { administered) }\end{array}$ & Sample size (response rate) & Participants (average age and/or age range) \\
\hline Anderson [37] & United States & $\begin{array}{l}\text { Survey (administered by the } \\
\text { American Medical Colleges and } \\
\text { the American College of } \\
\text { Obstetricians and Gynecologists) }\end{array}$ & $\begin{array}{l}<\text { Age 50, } 2000(40.3 \%) \\
>\text { Age 50, } 2100(57.3 \%)\end{array}$ & $\begin{array}{l}\text { Obstetrician-gynecologists (average age } \\
<50 \text { was } 44 \text { years, average age }>50 \text { was } \\
65 \text { years) }\end{array}$ \\
\hline Austrom [58] & United States & $\begin{array}{l}\text { Survey (modified version of } \\
\text { American Association of } \\
\text { Orthopaedic Surgeons survey) }\end{array}$ & $1834(43 \%)$ & $\begin{array}{l}\text { Multidisciplinary physicians and spouses } \\
\text { (average age } 75 \text { years) }\end{array}$ \\
\hline Baker [56] & United States & Survey & $500(46 \%)$ & Psychiatric physicians (age 50 to 69 years) \\
\hline Baker [59] & United States & Survey & $125(53 \%)$ & Black psychiatrists (age 31 to 74 years) \\
\hline Baker and Hishinuma [74] & United States & Survey & $\begin{array}{l}\text { AMA: } 187(58 \%) ; \\
\text { NMA: } 85(65 \%)\end{array}$ & $\begin{array}{l}\text { Multidisciplinary physicians. AMA } \\
\text { members (age } 50 \text { years or older), } \\
\text { NMA members (age } 30 \text { years or older) }\end{array}$ \\
\hline Batchelor [22] & United States & Survey/interviews & $20(80 \%)$ & Senior women physicians (age 59 to 95 years) \\
\hline Bieliauskas [75] & United States & Computerized cognitive test/survey & $359(82 \%)$ & $\begin{array}{l}\text { Surgeons (age } 45 \text { or older, average age } \\
61.4 \text { years) }\end{array}$ \\
\hline Brett [51] & Australia & Survey & $281(59 \%)$ & $\begin{array}{l}\text { Multidisciplinary physicians (age } 45 \text { to } 65 \text {, } \\
\text { average age } 52.4 \text { years) }\end{array}$ \\
\hline Burke [76] & United Kingdom & $\begin{array}{l}\text { Administrative data, Department } \\
\text { of Health and f } \\
\text { Insurance industry } \\
\text { (the Dentists' Provident Society) }\end{array}$ & 393(N/A) & Retired dentists (N/A) \\
\hline Chambers [69] & United Kingdom & Survey & $348(72 \%)$ & $\begin{array}{l}\text { Multidisciplinary physicians (average age } \\
55 \text { years) }\end{array}$ \\
\hline Crowson [6] & United States & $\begin{array}{l}\text { Retrospective study } \\
\text { (Duke University Hospital } \\
\text { Department of Human Resources) }\end{array}$ & 208 & $\begin{array}{l}\text { Multidisciplinary physicians (average age } \\
\text { between } 45 \text { and } 48.1 \text { ) }\end{array}$ \\
\hline Davidson [77] & United Kingdom & Survey & 2398 (78\%) & $\begin{array}{l}\text { Multidisciplinary physicians (average age } \\
\text { mid-40s) }\end{array}$ \\
\hline Davidson [52] & United Kingdom & Survey & 1460 (85\%) & $\begin{array}{l}\text { Multidisciplinary physicians (average age } \\
48 \text { years) }\end{array}$ \\
\hline Deitch [48] & United States & $\begin{array}{l}\text { Survey (ACR Committee } \\
\text { on Manpower) }\end{array}$ & 2804 (69\%) & $\begin{array}{l}\text { Radiologists, radio-oncologists, and } \\
\text { nuclear medicine specialists (average age } \\
\text { in years }<35(11 \%), 35 \text { and } 44(37 \%), 45 \\
\text { and } 54(32 \%) \text { and } 55 \text { or older }(20 \%) \text {. }\end{array}$ \\
\hline De Santo [78] & $\begin{array}{l}\text { Algeria, Australia, Brazil, } \\
\text { Egypt, France, Germany, } \\
\text { Greece, Italy, Malta, Libya, } \\
\text { Poland, Romania, } \\
\text { Slovak Republic, Slovenia, } \\
\text { Switzerland, The Netherlands, } \\
\text { Tunisia, Turkey, UK and USA }\end{array}$ & Survey & 113 (89.1\%) & $\begin{array}{l}\text { Active professors and emeritus/retired } \\
\text { professors from } 99 \text { departments of } \\
\text { medicine/universities worldwide (NA) }\end{array}$ \\
\hline
\end{tabular}


Table 1 Characteristics of included studies (Continued)

\begin{tabular}{|c|c|c|c|c|}
\hline Dodds [46] & United States & Survey & $96(82 \%)$ & $\begin{array}{l}\text { Academic chairs of ophthalmology } \\
\text { departments (age range }<50 \text { to }>70, \\
\text { average age } 58 \text { years) }\end{array}$ \\
\hline Donner [79] & United States & $\begin{array}{l}\text { Review of data based on survey } \\
\text { (ACR Commission on Human } \\
\text { Resources, } 2012 \text { and 2013) }\end{array}$ & N/A & Radiologists \\
\hline Draper [40] & Australia and New Zealand & Survey & $281(60 \%)$ & $\begin{array}{l}\text { Psychiatrists (ages } 55-87 \text { and average } \\
\text { age } 65.5 \text { years) }\end{array}$ \\
\hline Draper [80] & Australia and New Zealand & $\begin{array}{l}\text { Survey (respondents were fellows } \\
\text { of the Royal Australian and New } \\
\text { Zealand College of Psychiatrists } \\
\text { resident in Australia or New Zealand) }\end{array}$ & $529(57.9 \%)$ & Psychiatrists (age 40 years and older) \\
\hline Eagles [30] & United Kingdom & Survey & $180(50 \%)$ & Consultant psychologists (N/A) \\
\hline Evans and Ghosh [43] & United States & Survey & $749(17 \%)$ & Headache medicine specialists \\
\hline Farley [39] & United States & $\begin{array}{l}\text { Survey (American Academy of } \\
\text { Orthopaedic Surgeons in } \\
\text { cooperation with the Association } \\
\text { of American Medical Colleges } \\
\text { Center for Workforce Studies) }\end{array}$ & $3001(33.5 \%)$ & $\begin{array}{l}\text { Orthopedic surgeons (age } 50 \text { years } \\
\text { and older) }\end{array}$ \\
\hline $\begin{array}{l}\text { Fletcher and Schofield } \\
\text { [38] }\end{array}$ & Australia & $\begin{array}{l}\text { Data from the Australian Institute } \\
\text { of Health and Welfare (AlHW) } \\
\text { Medical Labour Force Survey } \\
\text { from } 1995 \text { to } 2003\end{array}$ & N/A & Psychiatrists (age 50 years and over) \\
\hline Florence [81] & United States & Survey & $785(22 \%)$ & $\begin{array}{l}\text { Transplant surgeons (average age } \\
48.7 \text { years) }\end{array}$ \\
\hline French [36] & United Kingdom & Survey & 2923(61\%) & $\begin{array}{l}\text { Consultants and specialists (average } \\
\text { age } 47 \text { years) }\end{array}$ \\
\hline French [23] & United Kingdom & Survey/interviews/focus groups & $924(50 \%)$ & $\begin{array}{l}\text { Multidisciplinary physicians (average } \\
\text { age } 43 \text { years) }\end{array}$ \\
\hline Gee [82] & United States & Telephone interview (Gallup Poll) & $451(89 \%)$ & $\begin{array}{l}\text { Urologists (age in years <36 (9\%), } 37 \\
\text { to } 45(29 \%), 46 \text { to } 54(30 \%), 55 \text { to } 64 \\
(25 \%),<65(7 \%))\end{array}$ \\
\hline Goldberg [57] & United States & $\begin{array}{l}\text { Survey of American College of } \\
\text { Emergency Physicians members } \\
\text { (two separate mailings in the fall } \\
\text { of } 2006 \text { and winter of 2007) }\end{array}$ & $1000(80 \%)$ & $\begin{array}{l}\text { American College of Emergency } \\
\text { Physicians members over the age of } \\
55 \text { years (average age } 57 \text { years) }\end{array}$ \\
\hline Grauer and Campbell [50] & Canada & Survey & $58(53.7 \%)$ & $\begin{array}{l}\text { Multidisciplinary physicians (average } \\
\text { age } 71.2 \text { years) }\end{array}$ \\
\hline $\begin{array}{l}\text { Greenfield and Proctor } \\
\text { [83] }\end{array}$ & United States & Survey & $659(75 \%)$ & $\begin{array}{l}\text { Surgeons (age in years <50 (7\%), } \\
50-60(29 \%), 60-70(35 \%),>70(28 \%)\end{array}$ \\
\hline Gregory and Menser, [63] & United States & $\begin{array}{l}\text { Longitudinal (three wave) } \\
\text { online survey }\end{array}$ & $\begin{array}{l}97,91,56(65.5 \%, 54.9 \% \\
58.4 \%), \text { respectively }\end{array}$ & Primary/ambulatory care physicians (N/A) \\
\hline Grondin [61] & Canada & Survey & 97 (71\%) & Thoracic surgeons (average age 47.7 years) \\
\hline
\end{tabular}


Table 1 Characteristics of included studies (Continued)

\begin{tabular}{|c|c|c|c|c|}
\hline Hall [5] & United States and Canada & Survey & $\begin{array}{l}1444 \text { APS members }(35 \%) ; \\
148 \text { pediatric department } \\
\text { chairs ( } 40 \%)\end{array}$ & $\begin{array}{l}\text { Senior pediatricians and pediatric } \\
\text { department chairs (ages } 39 \text { to } 94, \\
\text { average age } 65 \text { years) }\end{array}$ \\
\hline Heponiemi [44] & Finland & $\begin{array}{l}\text { Survey (Finnish Health Care } \\
\text { Professional Study) }\end{array}$ & $1393(27.9 \%)$ & $\begin{array}{l}\text { Multidisciplinary physicians } \\
\text { (ages } 45 \text { to } 65 \text { years) }\end{array}$ \\
\hline Hill [24] & United Kingdom & Semi-structured interviews/survey & $23(N / A)$ & Dentists (NA) \\
\hline Jacobson and Eran [25] & Israel & Interview & $317(89.5 \%)$ & $\begin{array}{l}\text { Multidisciplinary physicians } \\
\text { (age } 50 \text { years or older) }\end{array}$ \\
\hline $\begin{array}{l}\text { Jonasson and Kwakwa } \\
\text { [84] }\end{array}$ & United States & Survey & $373(84 \%)$ & General surgeons (NA) \\
\hline Joyce [42] & Australia & $\begin{array}{l}\text { Longitudinal survey (Medicine in } \\
\text { Australia: Balancing Employment } \\
\text { and Life, Cycles } 2009 \text { to 2012) }\end{array}$ & $\begin{array}{l}1073(2009,82.64 \%, 2010 \\
82.24 \%, 2011,75.51 \% \text { and } 2012,75.32 \%)\end{array}$ & Physicians and specialists aged $\geq 65$ years \\
\hline Kendell and Pearce [85] & United Kingdom & Survey & $173(82 \%)$ & Consultant psychiatrists (NA) \\
\hline Landon [49] & United States & $\begin{array}{l}\text { Data for this study are from } \\
\text { the first } 2 \text { rounds of the } \\
\text { Community Tracking Study } \\
\text { (CTS) Physician Survey }\end{array}$ & $16,681(63 \%)$ & $\begin{array}{l}\text { Primary care and specialist physicians } \\
\text { initially spending at least } 20 \mathrm{~h} / \text { week in } \\
\text { direct patient care activities were studied } \\
\text { (average age } 47.5 \text { years for practicing and } \\
63.0 \text { years for retired physicians) }\end{array}$ \\
\hline Lee [86] & United States & Telephone interview/survey & $33(75 \%)$ & $\begin{array}{l}\text { Multidisciplinary rural physicians } \\
\text { (age } 60 \text { years or older) }\end{array}$ \\
\hline Lee [87] & United States & Survey & $995(\mathrm{~N} / \mathrm{A})$ & $\begin{array}{l}\text { Surgeons (age in years }<35(13.37 \%) \text {, } \\
35-44(12.96 \%), 45-54(18.69 \%), 55-65 \\
(31.06 \%),>65(23.92 \%))\end{array}$ \\
\hline Luce [7] & United Kingdom & Survey & $518(72.5 \%)$ & $\begin{array}{l}\text { Multidisciplinary physicians } \\
\text { (age } 45 \text { years or older) }\end{array}$ \\
\hline Moriarty [88] & United States & $\begin{array}{l}\text { Survey sent to all members of } \\
\text { the American College of Radiology } \\
\text { (ACR), the Association of } \\
\text { University Radiologists (AUR), } \\
\text { and the Society of Chairs of } \\
\text { Academic Radiology Departments } \\
\text { (SCARD) }\end{array}$ & 37900 (11\%) & Practicing radiologists (NA) \\
\hline McGuirt and McGuirt [89] & United States & Survey & 438 (31.5\%) & $\begin{array}{l}\text { Otolaryngologists (ages } 40 \text { to } 80, \\
\text { average age } 63.2 \text { years) }\end{array}$ \\
\hline Mears [41] & United Kingdom & Survey & 835 (59\%) & $\begin{array}{l}\text { Consultant psychologists } \\
\text { (age } 50 \text { years or older) }\end{array}$ \\
\hline Newton [26] & United Kingdom & Semi-structured interviews & $21(N / A)$ & $\begin{array}{l}\text { Multidisciplinary physicians } \\
\text { (age } 44 \text { years or older) }\end{array}$ \\
\hline
\end{tabular}


Table 1 Characteristics of included studies (Continued)

\begin{tabular}{|c|c|c|c|c|}
\hline Onyura [19] & Canada & $\begin{array}{l}\text { Secondary analysis of data from a } \\
\text { larger study on issues of } \\
\text { late-career planning among } \\
\text { academic physicians; semi-structured } \\
\text { interviews }\end{array}$ & 21 & $\begin{array}{l}\text { Academic physicians at a Canadian } \\
\text { medical school }(n=21 \text {, average age }= \\
63 \text { years, age range }=46-72 \text { years })\end{array}$ \\
\hline Orkin [34] & United States & Survey & $8670(37.2 \%)$ & $\begin{array}{l}\text { Anaesthesiologists (age } 50-79 \text { years, } \\
\text { average age } 60.1 \text { years) }\end{array}$ \\
\hline Peisah [45] & $\begin{array}{l}\text { Australia, Canada, United } \\
\text { States }\end{array}$ & Semi-structured interviews & $25(\mathrm{~N} / \mathrm{A})$ & $\begin{array}{l}\text { Multidisciplinary physicians } \\
\text { (aged } 60 \text { or older, average age } 67.5 \text { years, } \\
\text { age range }=60-88 \text { years) }\end{array}$ \\
\hline Pit and Hansen [16] & Australia & Survey & $92(56 \%)$ & $\begin{array}{l}\text { Multidisciplinary physicians } \\
\text { (average age } 51 \text { years) }\end{array}$ \\
\hline Quandango [27] & United States & Semi-structured interviews & $40(\mathrm{~N} / \mathrm{A})$ & Multidisciplinary physicians (ages 55 to 72 ) \\
\hline Rayburn [31] & United States & $\begin{array}{l}\text { American Medical Association } \\
\text { Master file }\end{array}$ & N/A & Obstetrician-gynecologists \\
\hline Reuben and Silliman [47] & United States & Survey & $282(70 \%)$ & $\begin{array}{l}\text { Multidisciplinary physicians } \\
\text { (age } 65 \text { or older, average age } 71 \text { years) }\end{array}$ \\
\hline Rittenhouse [33] & United States & Survey & $967(\mathrm{~N} / \mathrm{A})$ & $\begin{array}{l}\text { Multidisciplinary physicians ( }<55 \text { years, } \\
62.8 \%, 55-64 \text { years, } 27.3 \%,>65 \text { years, } 9.9 \% \text { ) }\end{array}$ \\
\hline Rowe [90] & United States & Survey & $169(84 \%)$ & Physicians ( $52-96$ years) \\
\hline Sansom [28] & England & Semi-structured interviews & 23 & General practitioners (50-60 years) \\
\hline Shanafelt [53] & United States & $\begin{array}{l}\text { Survey, American Society of } \\
\text { Clinical Oncology }\end{array}$ & $2998(49.7 \%)$ & US oncologists \\
\hline Sibbald [32] & United Kingdom & Survey & 1949 (N/A) & $\begin{array}{l}\text { Multidisciplinary physicians } \\
\text { (average age } 55 \text { years) }\end{array}$ \\
\hline Silver [29] & Canada & Focus groups & 16 & $\begin{array}{l}\text { Academic physicians over } 50 \text { years old } \\
\text { within the Department of Medicine at } \\
\text { the University of Toronto }\end{array}$ \\
\hline Smith [91] & Canada & $\begin{array}{l}\text { National survey was administered } \\
\text { to all Canadian otolaryngologists }\end{array}$ & $65(65 \%)$ & $\begin{array}{l}\text { Otolaryngologists who were identified to } \\
\text { have a clinical practice composed of } \\
>50 \% \text { rhinology (average age: } 46 \text { years) }\end{array}$ \\
\hline Sutinen [35] & Finland & Survey & $819(55 \%)$ & $\begin{array}{l}\text { Multidisciplinary physicians } \\
\text { (ages } 26 \text { to } 63 \text { years) }\end{array}$ \\
\hline Van Greuningen [17] & Netherlands & Retrospective survey (2 waves) & $520(60 \%) ; 405(54 \%)$ & $\begin{array}{l}\text { Self-employed general practitioners } \\
\text { retired before age } 65\end{array}$ \\
\hline Wakeford et al. [18] & United Kingdom & Interview & $250(79 \%)$ & $\begin{array}{l}\text { Multidisciplinary physicians (average age: } \\
61.4 \text { years) }\end{array}$ \\
\hline
\end{tabular}


Table 2 Assessment of studies included in this review using the Newcastle-Ottawa Quality Assessment Scale for cohort studies as well as the adapted version for cross-sectional studies

\begin{tabular}{|c|c|c|c|c|c|c|c|c|}
\hline \multirow[b]{2}{*}{37} & \multicolumn{4}{|l|}{ Selection $^{a}$} & \multirow[t]{2}{*}{ Comparability ${ }^{b}$} & \multicolumn{2}{|l|}{ Outcome $^{c}$} & \multirow{2}{*}{$\begin{array}{l}\text { Quality } \\
\text { score }\end{array}$} \\
\hline & $\begin{array}{l}\text { Representativeness of } \\
\text { sample }\end{array}$ & $\begin{array}{l}\text { Sample } \\
\text { size }\end{array}$ & $\begin{array}{l}\text { Non- } \\
\text { respondents }\end{array}$ & $\begin{array}{l}\text { Ascertainment of } \\
\text { exposure }\end{array}$ & & $\begin{array}{l}\text { Assessment of } \\
\text { outcome }\end{array}$ & $\begin{array}{l}\text { Statistical } \\
\text { test }\end{array}$ & \\
\hline Anderson [47] & A & A & $B$ & C & A & C & $A$ & 6 \\
\hline Austrom [58] & B & A & A & B & - & C & A & 6 \\
\hline Baker [56] & A & A & A & C & - & C & B & 4 \\
\hline Baker [59] & A & A & A & B & - & C & B & 5 \\
\hline $\begin{array}{l}\text { Baker and } \\
\text { Hishinuma [74] }\end{array}$ & B & A & B & B & $A / B$ & C & A & 7 \\
\hline Batchelor [22] & C & B & B & B & - & C & B & 2 \\
\hline Biellauskas [75] & B & B & C & A & $\mathrm{A} / \mathrm{B}$ & C & A & 6 \\
\hline Brett [51] & B & B & B & B & - & A & A & 5 \\
\hline Burke [76] & C & A & C & B & - & C & B & 3 \\
\hline Chambers [69] & A & A & A & A & - & C & A & 6 \\
\hline Crowson, [6] & A & A & A & A & A & B & - & 7 \\
\hline Davidson [77] & A & A & A & C & - & C & A & 5 \\
\hline Davidson [52] & A & A & C & B & A & C & A & 6 \\
\hline Deitch [48] & A & A & A & B & $A / B$ & C & A & 8 \\
\hline De Santo [78] & A & A & B & B & - & C & B & 4 \\
\hline Dodds [46] & A & A & A & A & $A / B$ & C & A & 9 \\
\hline Donner [79] & $\mathrm{D}$ & C & C & C & - & $\mathrm{D}$ & B & 0 \\
\hline Draper [40] & A & A & A & B & A & $C$ & A & 7 \\
\hline Draper [80] & A & A & B & A & $A / B$ & C & A & 8 \\
\hline Eagles [30] & A & A & B & B & A & C & B & 5 \\
\hline $\begin{array}{l}\text { Evans and Ghosh } \\
\text { [43] }\end{array}$ & A & B & B & A & - & C & A & 5 \\
\hline Farley [39] & A & A & B & A & - & C & B & 4 \\
\hline $\begin{array}{l}\text { Fletcher and } \\
\text { Schofield [38] }\end{array}$ & A & A & C & A & $\mathrm{A} / \mathrm{B}$ & C & A & 8 \\
\hline Florence [81] & A & A & B & B & - & C & B & 4 \\
\hline French [36] & A & A & A & A & A & C & A & 8 \\
\hline French [23] & A & A & A & A & A & C & A & 8 \\
\hline Gee [82] & A & A & B & B & - & C & A & 5 \\
\hline Goldberg [57] & A & A & B & A & - & C & A & 6 \\
\hline $\begin{array}{l}\text { Grauer and } \\
\text { Campbell [50] }\end{array}$ & $\mathrm{D}$ & B & C & B & - & C & B & 2 \\
\hline $\begin{array}{l}\text { Greenfield and } \\
\text { Proctor [83] }\end{array}$ & A & A & B & B & A & $C$ & B & 5 \\
\hline $\begin{array}{l}\text { Gregory and } \\
\text { Menser [63] }\end{array}$ & B & A & B & A & A & C & A & 7 \\
\hline Grondin [61] & A & A & B & A & - & C & A & 6 \\
\hline Hall [5] & A & A & B & B & - & C & B & 4 \\
\hline Heponiemi [44] & A & A & B & A & $A / B$ & C & A & 8 \\
\hline Hill [24] & C & A & C & B & - & C & B & 3 \\
\hline $\begin{array}{l}\text { Jacobson and Eran } \\
\text { [25] }\end{array}$ & A & A & B & A & $A / B$ & C & A & 8 \\
\hline $\begin{array}{l}\text { Jonasson and } \\
\text { Kwakwa [84] }\end{array}$ & A & A & B & B & A & C & B & 5 \\
\hline
\end{tabular}


Table 2 Assessment of studies included in this review using the Newcastle-Ottawa Quality Assessment Scale for cohort studies as well as the adapted version for cross-sectional studies (Continued)

\begin{tabular}{|c|c|c|c|c|c|c|c|c|}
\hline Joyce [42] & $A$ & $A$ & A & C & A & C & B & 8 \\
\hline $\begin{array}{l}\text { Kendell and Pearce } \\
{[85]}\end{array}$ & A & A & B & C & - & $C$ & B & 3 \\
\hline Landon [49] & $\mathrm{B}$ & A & A & B & $\mathrm{A} / \mathrm{B}$ & $C$ & A & 8 \\
\hline Lee [77] & A & A & A & B & - & $C$ & B & 5 \\
\hline Lee [87] & $B$ & A & B & B & - & $C$ & A & 5 \\
\hline Luce [7] & A & A & B & A & - & C & A & 6 \\
\hline Moriarty [88] & A & $B$ & B & B & $\mathrm{A} / \mathrm{B}$ & $C$ & B & 5 \\
\hline $\begin{array}{l}\text { McGuirt and } \\
\text { McGuirt [89] }\end{array}$ & B & A & B & B & - & $C$ & B & 4 \\
\hline Mears [41] & A & A & B & B & A & $C$ & A & 6 \\
\hline $\begin{array}{l}\text { Meghea and } \\
\text { Sunshine [54] }\end{array}$ & A & A & A & B & $A / B$ & $C$ & A & 8 \\
\hline Newton [26] & $C$ & A & B & A & - & $C$ & B & 4 \\
\hline Onyura [19] & $\mathrm{B}$ & A & C & B & - & C & B & 4 \\
\hline Orkin [34] & A & A & B & B & $\mathrm{A} / \mathrm{B}$ & $C$ & A & 7 \\
\hline Peisah [45] & $C$ & A & C & A & - & $C$ & B & 4 \\
\hline Pit and Hansen [16] & $\mathrm{B}$ & A & B & A & $\mathrm{A} / \mathrm{B}$ & $C$ & A & 8 \\
\hline Quandango [27] & C & A & B & B & - & C & B & 3 \\
\hline Rayburn [31] & A & A & B & B & - & $\mathrm{B}$ & B & 5 \\
\hline $\begin{array}{l}\text { Reuben and } \\
\text { Silliman [47] }\end{array}$ & A & A & A & B & $\mathrm{A} / \mathrm{B}$ & C & A & 8 \\
\hline Ritternhouse [33] & A & A & A & $\mathrm{B}$ & $\mathrm{A} / \mathrm{B}$ & $\mathrm{B}$ & A & 9 \\
\hline Rowe [90] & A & A & B & C & - & C & B & 3 \\
\hline Shanafelt [53] & A & A & A & A & $\mathrm{A} / \mathrm{B}$ & $C$ & A & 9 \\
\hline Sibbald [32] & A & A & A & A & $A / B$ & A & A & 9 \\
\hline Silver [29] & $\mathrm{B}$ & A & B & B & - & C & B & 4 \\
\hline Smith [91] & A & A & C & A & - & C & B & 5 \\
\hline Sutinen [35] & A & A & A & A & $A / B$ & C & A & 8 \\
\hline $\begin{array}{l}\text { Van Greuningen } \\
{[17]}\end{array}$ & A & A & B & A & - & $C$ & A & 7 \\
\hline Wakeford [18] & A & A & C & B & - & C & B & 4 \\
\hline
\end{tabular}

"-", not reported.

Wells, G.A.; Shea, B.; O'Connell, D.; Peterson, J.; Welch, V.; Losos, M.; Tugwell, P. The Newcastle-Ottawa Scale (NOS) for assessing the quality of nonrandomized studies in meta-analyses. Available online: http://www.ohri.ca/programs/clinical_epidemiology/oxford.asp

${ }^{a}$ Selection (5 points in total): (1) Representativeness of the sample: A, truly representative of the average in the target population (1 point); $\mathrm{B}$, somewhat representative of the average in the target population (1 point); $C$, selected group of users (no points); $D$, no description of the sampling strategy (no points). (2) Sample size: A, justified and satisfactory (1 point); B, not justified (no points). (3) Non-respondents: A, comparability between respondents and non-respondents characteristics is established, and the response rate is satisfactory ( 1 point); $B$, the response rate is unsatisfactory, or the comparability between respondents and non-respondents is unsatisfactory (no points); $C$, no description of the response rate or the characteristics of the responders and the non-responders (no points). (4) Ascertainment of the exposure: A, validated measurement tool (2 points); B, non-validated measurement tool, but the tool is available or described (1 point); $\mathrm{C}$ no description of the measurement tool (no points).

${ }^{b}$ Comparability ( 2 points in total): (1) Confounding factors are controlled: A, the study controls for the most important factor (1 point); B, the study control for any additional factor (1 point).

c Outcome (3 points in total): (1) Assessment of the outcome: A, independent blind assessment (2 points); B, record linkage (2 points); C, self-report (1 point); D, no description (no points). (2) Statistical test: $A$, the statistical test used to analyze the data is clearly described and appropriate, and the measurement of the association is presented, including confidence intervals and the probability level ( $P$ value) (1 point); B, the statistical test is not appropriate, not described or incomplete (no points).

were associated with intentions to retire [28, 43]. Medicolegal issues often arose from a lack of satisfaction with the regulation of medicine for reasons of unwelcome change, bureaucracy, oppressive management [26, 35, 44], and issues with physician partners [26, 45]. Experiencing poor health, cognitive decline, difficulty sleeping, and psychological distress were also factors leading to a physician's retirement $[15,18,19,34,36,38,46-50]$. 
Table 3 Assessment of qualitative studies included in this review

\begin{tabular}{|c|c|c|c|c|c|c|c|c|c|c|}
\hline & $\begin{array}{l}\text { Batchelor, } \\
1990[22]\end{array}$ & $\begin{array}{l}\text { French } \\
\text { et al., } \\
2006 \\
{[23]}\end{array}$ & $\begin{array}{l}\text { Hill } \\
\text { et al., } \\
2010\end{array}$ & $\begin{array}{l}\text { Jacobson } \\
\text { and Eran, } \\
1980[24]\end{array}$ & $\begin{array}{l}\text { Newton } \\
\text { et al., } \\
2004 \\
{[26]}\end{array}$ & $\begin{array}{l}\text { Peisah, } \\
\text { Gautam, } \\
\text { and } \\
\text { Goldstein, } \\
2009[9]\end{array}$ & $\begin{array}{l}\text { Quandango, } \\
1978[27]\end{array}$ & $\begin{array}{l}\text { Sansom, } \\
2016 \\
{[28]}\end{array}$ & $\begin{array}{l}\text { Silver, } \\
\text { Pang, } \\
\text { and } \\
\text { Williams, } \\
2015 \text { [29] }\end{array}$ & $\begin{array}{l}\text { Wakeford, } \\
\text { Roden, and } \\
\text { Rothman, } \\
1986[18]\end{array}$ \\
\hline $\begin{array}{l}\text { 1. Does the study address a clearly } \\
\text { focused question/issue? }\end{array}$ & $\mathrm{Y}$ & Y & $\mathrm{Y}$ & $\mathrm{Y}$ & $\mathrm{Y}$ & $\mathrm{Y}$ & $\mathrm{Y}$ & $\mathrm{Y}$ & Y & Y \\
\hline $\begin{array}{l}\text { 2. Is the research method (study } \\
\text { design) appropriate for answering } \\
\text { the research question? }\end{array}$ & Y & Y & Y & Y & Y & Y & Y & Y & Y & Y \\
\hline 3. Was the context clearly described? & Y & Y & Y & Y & Y & Y & Y & Y & Y & Y \\
\hline $\begin{array}{l}\text { 4. How was the fieldwork undertaken? } \\
\text { Was it described in detail? Are the } \\
\text { methods for collecting data clearly } \\
\text { described? }\end{array}$ & Y & Y & Y & Y & Y & N & Y & Y & Y & N \\
\hline $\begin{array}{l}\text { 5. Could the evidence (fieldwork } \\
\text { notes, interview transcripts, } \\
\text { recordings, documentary analysis, } \\
\text { etc.) be inspected independently by } \\
\text { others? }\end{array}$ & N & Y & Y & N & Y & Y & Y & Y & Y & N \\
\hline $\begin{array}{l}\text { 6. Are the procedures for data analysis } \\
\text { reliable and theoretically justified? } \\
\text { Are quality control measures used? }\end{array}$ & $\mathrm{N}$ & Y & Y & N & Y & $\mathrm{N}$ & Y & Y & Y & N \\
\hline $\begin{array}{l}\text { 7. Was the analysis repeated by more } \\
\text { than one researcher to ensure } \\
\text { reliability? }\end{array}$ & $N$ & $Y$ & $Y$ & $Y$ & $Y$ & $\mathrm{~N}$ & $N$ & $Y$ & $Y$ & $\mathrm{~N}$ \\
\hline $\begin{array}{l}\text { 8. Are the results credible, and if so, } \\
\text { are they relevant for practice? }\end{array}$ & $Y$ & $Y$ & $Y$ & $Y$ & $Y$ & $\mathrm{~N}$ & $Y$ & $Y$ & $Y$ & $Y$ \\
\hline $\begin{array}{l}\text { 9. Are the conclusions drawn justified } \\
\text { by the results? }\end{array}$ & $Y$ & $Y$ & $Y$ & $Y$ & $Y$ & $Y$ & $Y$ & $Y$ & $Y$ & $Y$ \\
\hline $\begin{array}{l}\text { 10. Are the findings of the study } \\
\text { transferable to other settings? }\end{array}$ & $Y$ & $\mathrm{~N}$ & $Y$ & $Y$ & $Y$ & $\mathrm{~N}$ & $Y$ & $Y$ & $\mathrm{~N}$ & $\mathrm{~N}$ \\
\hline
\end{tabular}

Responses in the affirmative $(\mathrm{Y})$ are indicative of higher validity and quality; those in the negative $(\mathrm{N})$ indicate absence of support.

Adapted from Crombie, The Pocket Guide to Critical Appraisal; the critical appraisal approach used by the Oxford Centre for Evidence Medicine, checklists of the

Dutch Cochrane Centre, BMJ editor's checklists and the checklists of the EPPI Centre.

The decision to retire early was also linked to preserving one's health to lead a healthy retirement $[51,52]$. Financial issues contributing to a physician's early retirement included: increasing costs of retaining a practice, malpractice costs, and other economic pressures [5, 25, 37, 39, 47, 52], insufficient financial remuneration, and pension security [7, 46, 52, 53]. However, one study [42] found that retirement was not associated with perceived adequacy of finances, or general health status. Several studies noted that physicians working in institutions or in countries where the policy landscape changed considerably were more inclined to retire in part due to poor work satisfaction that resulted from changing circumstances around the delivery of care and doctoring regulations [29]. Table 5 summarizes the obstacles related to continuing practice.

\section{Reasons for delaying retirement}

Reasons for physicians delaying retirement included being satisfied with their career [16, 34, 37, 39, 47-49], institutional flexibility [51], a feeling of responsibility for their patients $[18,19,37,38,47,51]$, a desire to be healthy and keep being active [18, 34, 44, 46], financial reasons $[7,34,36,39,46,47,50,53,54]$, and a lack of interests outside of medicine [46]. In particular, institutional flexibility was a positive driver of physicians' work satisfaction and their desire to remain in practice as they were provided reasonable access to sabbaticals, flexible working hours, and control over their job and career development [7, 39, 51, 55].

The continuation of medical practice is deeply rooted in a desire to keep active and focus on the social and intellectual elements of continuing to practice [46, 47, 56]. Physicians expressed concerns over their decision to retire, due to fear of losing their primary identity or purpose $[9,19,50,57]$, or being uncomfortable with the methods used to enforce their retirement [58]. Retirement concerns also stemmed from personal issues such as a fear of potential changes in the relationship with their spouse following retirement [58], a fear of excessive leisure time 
Table 4 Expected and actual physician retirement age

\begin{tabular}{|c|c|c|c|c|}
\hline & $50-59$ years & $60-69$ years & $>70$ years & "Never" \\
\hline Expected retirement age & $\begin{array}{l}\text { Burke [76] } \\
\text { Eagles [30] } \\
\text { Luce [7] } \\
\text { Fletcher [38] } \\
\text { Mears [41] } \\
\text { Goldberg [57] } \\
\text { Sansom [28] }\end{array}$ & $\begin{array}{l}\text { Anderson [37] } \\
\text { Dietch [48] } \\
\text { Dodds [46] } \\
\text { Farley [39] } \\
\text { Florence [81] } \\
\text { Grondin [61] } \\
\text { Mears [41] } \\
\text { French [36] } \\
\text { French [23] } \\
\text { Gee [82] } \\
\text { Pit [45] } \\
\text { Rayburn [31] } \\
\text { Shanafelt [53] } \\
\text { Smith [91] } \\
\text { Wakeford [18] }\end{array}$ & Batchelor [22] & $\overline{\text { Draper [40] }}$ \\
\hline Actual retirement age & $\begin{array}{l}\text { Baker [52] } \\
\text { Eagles [30] } \\
\text { Sansom [28] }\end{array}$ & $\begin{array}{l}\text { Anderson [37] } \\
\text { Austrom [58] } \\
\text { Batchelor [22] } \\
\text { Farley [39] } \\
\text { Fletcher [38] } \\
\text { French [36] } \\
\text { Jonasson [84] } \\
\text { Meghea [54] } \\
\text { Luce [7] } \\
\text { Orkin [34] } \\
\text { Rayburn [31] } \\
\text { Rowe [90] } \\
\text { Van Greuningen [17] } \\
\text { Wakeford [18] }\end{array}$ & $\begin{array}{l}\text { Joyce [42] } \\
\text { Rayburn [31] }\end{array}$ & - \\
\hline
\end{tabular}

Note: Average or highest reported retirement ages are reported.

Studies where the majority of physicians met retirement age expectations are in italics

Table 5 Obstacles to practice

\begin{tabular}{|c|c|}
\hline Subtheme & Study \\
\hline $\begin{array}{l}\text { Workplace frustration: bureaucracy, accreditation, } \\
\text { healthcare reform, demands from government, } \\
\text { alienation by changes to working life, low job control, } \\
\text { low organizational justice, poor teamwork and } \\
\text { workforce shortages }\end{array}$ & $\begin{array}{l}\text { Brett [51]; Crowson [6]; Evans [43]; Fletcher } \\
\text { and Schofield [38]; Heponiemi [44]; Hill [24]; } \\
\text { Kendell and Pearce [85]; Lee [87]; McGuirt } \\
\text { and McGuirt [89]; Mears [41]; Newton [26]; } \\
\text { Sansom [23]; Sutinen [35]; Van Greuningen [17] }\end{array}$ \\
\hline $\begin{array}{l}\text { Workload pressures: patient demands, long hours, } \\
\text { demanding on-call schedules and sacrifice of } \\
\text { family/free time, work-life balance }\end{array}$ & $\begin{array}{l}\text { Brett [51]; Chambers [69]; Draper [80]; Evans [43]; } \\
\text { French [36]; Joyce [42]; Mears [41]; Meghea } \\
\text { and Sunshine 549]; Newton [26]; Goldberg [57]; } \\
\text { Sibbald [32]; Onyura [19]; Sansom [28]; Shanafelt [53]; } \\
\text { Van Greuningen [17] }\end{array}$ \\
\hline Career dissatisfaction: lost interest in work & $\begin{array}{l}\text { Brett [51]; Chambers [69]; Crowson [6]; Evans [43]; } \\
\text { Hill [24]; Joyce [42]; Luce [7]; Orkin [34]; } \\
\text { Ritternhouse [33]; Sibbald [32]; Landon [49]; } \\
\text { Van Greuningen [17] }\end{array}$ \\
\hline $\begin{array}{l}\text { Health: excessive stress, health and mental health } \\
\text { concerns (thoughts of suicide, emotional exhaustion), } \\
\text { and spousal health }\end{array}$ & $\begin{array}{l}\text { Dodds [35]; Draper [80]; Goldberg [57]; Hall [5]; } \\
\text { Hill [24]; Luce [7]; Newton [26]; Pit and Hansen [16]; } \\
\text { Sansom [28]; Van Greuningen [17] }\end{array}$ \\
\hline $\begin{array}{l}\text { Finances: pension, economic concerns, costs of } \\
\text { continuing to practice, retirement not being } \\
\text { written into partner agreements, general guidance, } \\
\text { insurer policies affecting payment }\end{array}$ & $\begin{array}{l}\text { Evans [43]; French [36]; Grondin [75]; Hall [5]; Lee [78]; } \\
\text { Orkin [34]; Sansom [28]; Van Greuningen [17]; Wakeford [18] }\end{array}$ \\
\hline $\begin{array}{l}\text { Skills and competencies: worry over competencies amidst technological advancements } \\
\text { and new modalities of diagnosis or treatment }\end{array}$ & $\begin{array}{l}\text { Crowson [6]; Draper [80]; Goldberg [57]; Grauer and } \\
\text { Campbell [50]; Hall [5]; Sansom [28] }\end{array}$ \\
\hline
\end{tabular}


and lack of hobbies [50], and inadequate financial preparation for retired life [34, 57]. Several studies also pointed to a link between physicians' restricted availability of free time and the development of external hobbies or interests. Nonetheless, continuing in medicine was viewed as a better alternative to life in retirement $[52,56,59]$.

\section{Strategies to facilitate physician retention and retirement planning}

Key strategies to facilitate physician retention and retirement planning included offering flexible work hours, minimal work barriers, enhancing work satisfaction, prioritizing physician health, and attention to finances. In particular, options such as part-time employment and less bureaucracy were suggested as ways to facilitate a working environment that would be amenable to physicians overburdened by work demands in ways that might foreshorten their career. In addition, providing opportunities for professional development to help physicians develop or change the content of their work was offered as an important means of retention, as well as a mechanism for making successful later career transitions out of medicine. Attention to personal matters such as physicians' own health and finances in ways that reduced work-related stress or protected physicians' income through pension plans were also important in enhancing physician retention and enticing continued practice. Table 6 summarizes the retention schemes described by the studies included in this review.

\section{Discussion}

Our review confirmed that physicians are likely to remain in their practice beyond the traditional retirement age of 65 . To put these results into context, it is worthwhile to first consider that in recent decades, workers are generally tending toward later retirement. While a person aged 50 in the workforce during 1997 was expected to continue working 13 more years, an average worker of the same age in 2009 was expected to work an additional 3 years, eventually retiring at an age of 66 on average [60]. This systematic review illustrates that the average age physicians expect to retire lies closest to age 60 while their age at actual retirement is closer to 69 . This represents an average of 3 years later than the general population.

Retirement trends have been shifting over the last few decades in response to an increasing lifespan, adjustments to economic market fluctuations, and concerns about the sustainability of social security entitlements [61, 62]. In particular, concerns about economic market fluctuations are particularly relevant for physicians who tend not to have access to group pension funds that other workers, such as teachers or health-care administrators, might have access to. We found delayed retirement among physicians is likely to be influenced by flexibility and intensity of working hours, work satisfaction, career opportunities, resource adequacy, intrinsic value, convenience, financial incentives, and relations with co-workers. As one might expect, these are many of the same determinants that impact retirement among other professionals. However, it is also likely that other factors such as attachment to work and strong work identity may serve as an additional rationale for working beyond the traditional retirement age [29]. Furthermore, it is likely that the advanced training and late entry into the work force also renders physicians more likely to retire later than the average worker.

Physician's early retirement, like that of other professions, is often brought about by negative dimensions of work satisfaction. Where physicians may differ from the general population is in the complex nature of their work, which involves a unique combination of advanced training, autonomy, skill, experience, leadership, and decision-making that can have life or death consequences. Many studies have examined the implications

Table 6 Retention schemes

\begin{tabular}{|c|c|}
\hline Subtheme & Study \\
\hline $\begin{array}{l}\text { Flexible work hours: part-time employment options, } \\
\text { gradual reduction, flexible hours or sabbatical, } \\
\text { decreased on-call, relief of workload pressure }\end{array}$ & $\begin{array}{l}\text { Anderson [37]; Brett [51]; Davidson [52]; Eagles [30]; } \\
\text { French [36]; French [23]; Hall [5]; Jacobsen and Eran [25]; } \\
\text { Newton [26], 2004; Goldberg [57] }\end{array}$ \\
\hline $\begin{array}{l}\text { Minimal work barriers: less bureaucracy, increased staff, } \\
\text { improved working conditions, support to } \\
\text { maintain/update competencies, more time with patients }\end{array}$ & Brett [51]; Davidson [52]; Eagles [30]; Kendell and Pearce [85] \\
\hline $\begin{array}{l}\text { Work satisfaction: professional/clinical freedom, } \\
\text { attend conferences and rounds, office space, } \\
\text { chances to develop or change content of their } \\
\text { work (i.e., teaching opportunities) }\end{array}$ & Brett [51]; Chambers [69]; Eagles [30]; Farley [39]; Hall [5]; Landon [49] \\
\hline $\begin{array}{l}\text { Health: continuing good or better than expected } \\
\text { health at expected retirement age, strategies to } \\
\text { reduce work-related stress, support prioritizing health }\end{array}$ & Brett [51]; Davidson [52]; Draper [80]; Luce [7]; Pit and Hansen [16] \\
\hline Finances: protected pensions, being highly paid, financial necessity & Brett [51]; Davidson [52]; Eagles [30]; French [36]; Hall [5]; \\
\hline
\end{tabular}


of physician burnout $[43,63]$, thus suggesting that physicians face unique challenges as it relates to extending their careers. Physicians' early retirement is an important concern as other research attests to the risks to patient care associated with physician shortages [64]. While the studies examined in this review did not highlight gender as an important factor relevant to early retirement, there is evidence to indicate that there are high burnout rates among women physician [65]. This is likely to influence physician retirement rates in ways that were not captured in the studies reviewed here, and relevant given than women live longer than men on average and are increasingly entering medicine.

Successful retirement planning was found to be related to being prepared for the financial demands, physical changes, and psychosocial dynamics associated with aging and leaving the workforce, consistent with prior research based on the general population [66-68]. Findings based on these studies of physicians suggest that a reduction of working hours may present as one of the most successful instruments for staff retention [17]. A shift toward non-clinical duties such as teaching and mentorship may also help with retention [42] and also facilitate knowledge transfer to younger professionals. The theory of purposeful work behavior [59] posits that, when job characteristics act in concert with individuals' motivational striving, psychological meaningfulness may be gleaned from their work. Thus, if physicians are given opportunities to pursue preferred work tasks such as teaching over clinical rounds $[30,69]$, then their experiences of greater meaningfulness in their work may trigger task-specific motivation [70]. This can result in a willingness to continue working in hospital settings in a way that benefits the enterprise as a whole.

On the whole, health was also shown to be an important factor determining whether physicians chose to remain in the workforce. Excessive workload and poor health were found to be major reasons a physician may choose to retire. As such, healthcare organizations may consider strategies that improve physician health by addressing the physical fitness and risk-related habits of physicians. Some potential interventions might include fostering a culture that is supportive of taking sick days [71] along with proper mechanisms that allow physicians not to overburden one another when taking sick days. Findings from most of the studies included in this review also indicate that a supportive and highly satisfying work environment facilitates physician retention.

Organizations can have a role in facilitating the graceful and timely exit of the well-established physician but should exercise caution that the approach taken is not driven by ageist stereotypes or leading to feelings of being "pushed out" [26]. Physicians retiring beyond the traditional retirement age will have accumulated decades of knowledge and experience, and offer an invaluable resource to the medical enterprise [42]. The challenge is that, without foresight of the timing associated with physicians' plans for retirement, institutional hospital succession plans come to a halt. The timing of physician retirement becomes particularly salient, not only for human resources planning but also for patient care continuity and transitions of care in hospital enterprises where mentors of the younger hospitalist workforce may be scarce [3]. In this way, the medical enterprise must strike a delicate balance between encouraging preparation for retirement and delaying the timing and eventual transitions of its most experienced staff who will be replaced by a growing pool of younger physicians who stand waiting in the wings for professional opportunities.

\section{Limitations and recommendations for future research}

Research on the factors that influence physician retirement timing and planning for retirement is still in its early stages, and future exploration into the most promising interventions is needed to further delineate our preliminary findings. Some limitations of this review include the restriction to English language studies, which excludes the perspectives of physicians from nonEnglish speaking regions. Furthermore, our analysis is based on a heterogeneous sample of physicians spanning across diverse specializations, with jurisdictional differences in regulations, mandatory retirement legislation, pension systems, and differences in remuneration across healthcare systems. In addition, because the studies examined in this review used a cross-sectional design and were limited in terms of the types of analyses they performed, we were unable to perform a meta-analysis of the included studies. Furthermore, our search was restricted to peer-reviewed literature, thus future research may enhance the findings of this study by examining the grey literature on this topic. Future studies can also benefit from exploring the healthcare context in which the physicians practice, gender differences as they relate to physician retirement planning and physicians' transitions from practice, and consider following physicians over time to better understand factors that facilitate planning for a transition from practice.

The abolition of policies of mandatory retirement across many countries has encouraged some physicians to extend their medical careers, generating greater unpredictability in later career transitions [5, 31]. While several attempts, including our own study, have aimed at improving understanding of health workforce issues and implications of aging and timing of physicians' work, future policy research should continue forecasting physician retirement trajectories and human resource strategies in ways that can account for older physicians who want to remain in clinical practice beyond traditional 
retirement age [17, 72, 73]. Recommendations for next steps in policy reform at the organizational and health system level may come from within hospital and other related organizations which aim to address intentions to leave by improving psychosocial working conditions for the medical profession [64] and scaling back workloads to retain the best talent in experienced physicians [42]. Notably, healthcare managers may pursue recommendations for an "integrated" approach to recruitment, retention, and retirement planning that aids in better anticipating upcoming retirement transitions, shifts cultural attitudes toward retirement planning, and brings together a larger strategy to ameliorate succession planning.

\section{Conclusions}

Knowledge of when physicians plan to retire and how they can transition out of practice has been shown to aid effectual succession planning. This paper identified that the most common age of retirement for physicians was between 60 and 69 . We examined the literature on reasons for early and delayed retirement, as well as strategies shown to be effective in supporting continuing practice. We found excessive workload, poor health, and low job satisfaction to be major reasons for why a physician may choose to retire early. Delayed retirement or reasons physicians' work lives were extended was explained by financial obligations, strong work identity, career satisfaction, and institutional flexibility. Strategies that supported continuing to practice included offering flexible work hours, minimal work barriers, enhancing work satisfaction, prioritizing physician health, and attention to finances. As this line of inquiry is still developing, we recommend future research and strategies consider the impact of a physician's flexible work hours, gradual reduction in responsibilities, and the ways in which resources for financial planning facilitate physician retirement planning. In addition, an important component of successful retirement planning concerns the creation of meaningful activity after retirement [31]; thus, healthcare organizations should consider promoting retirement resource toolkits, education sessions, and guidance around financial planning for physicians throughout their careers, as well as creating post-retirement opportunities that maintain institutional ties through teaching, mentoring, and peer support $[68,69]$. Preparation for a retirement that is tailored to physicians' career stages and specific age can avoid the complications that arise when a physician's career trajectory does not correspond to his or her expectations or what is in the best interests of the medical practice plan.

\section{Acknowledgements}

Not applicable.

\section{Funding}

This work was supported by funding from the Mitacs-Accelerate Program. The funders had no role in study design, data collection or analysis, decision to publish, or preparation of the manuscript.

\section{Availability of data and materials \\ Not applicable.}

\section{Authors' contributions}

All authors contributed to the quality assessment and data analysis portions of the manuscript. MPS conceived of the study and led the overall study design. $\mathrm{ADH}$ held primary responsibility for the database searches and data extraction. $A B$ held primary responsibility for compiling the quality assessment. NIW held primary responsibility for assembling the thematic analysis. MPS, NIW, and AB contributed to the writing of the manuscript. All authors read and approved the final manuscript.

Competing interests

The authors declare that they have no competing interests.

Consent for publication

Not applicable.

Ethics approval and consent to participate

Not applicable.

Received: 20 April 2016 Accepted: 1 November 2016

Published online: 15 November 2016

\section{References}

1. Young P, Smith C, Pettigrew L, Seo H, Blane D. International work and leadership in UK general practice. Leadersh Health Serv. 2014;27(2):87-103.

2. AMA: Competency and retirement: Evaluating the senior physician. AMA Wire. 2015. http://www.ama-assn.org/ama/ama-wire/post/competencyretirement-evaluating-senior-physician. Accessed 26 July 2016.

3. Kneeland PP, Kneeland C, Wachter RM. Bleeding talent: a lesson from industry on embracing physician workforce challenges. J Hosp Med. 2010;5(5):306-10.

4. LoboPrabhu SM, Molinari VA, Hamilton JD, Lomax JW. The aging physician with cognitive impairment: approaches to oversight, prevention, and remediation. Am J Geriatr Psychiatry. 2009;17(6):445-54.

5. Hall JG. The challenge of developing career pathways for senior academic pediatricians. Pediatr Res. 2005;57(6):914-9.

6. Crowson MG, Vail C, Eapen RJ. Influence of electronic medical record implementation on provider retirement at a major academic medical centre. J Eval Clin Pract. 2015;22(2):222-7.

7. Luce A, van Zwanenberg T, Firth-Cozens J, Tinwell C. What might encourage later retirement among general practitioners? J Manag Med. 2002;16(4-5):303-10.

8. Silver MP, Hamilton AD, Biswas A, Williams SA. Life after medicine: a systematic review of studies of physicians' adjustment to retirement. Arch Community Med Public Health. 2016;1(1):26-32.

9. Peisah C, Gautam M, Goldstein MZ. Medical masters: a pilot study of adaptive ageing in physicians. Australas J Ageing. 2009;28(3):134-8.

10. Moher D, Liberati A, Tetzlaff J, Altman DG. Preferred reporting items for systematic reviews and meta-analyses: the PRISMA statement. Ann Intern Med. 2009;151(4):264-9.

11. Wells GA, Shea B, Peterson JEA, Welch V, Losos M, Tugwell P. The Newcastle-Ottawa Scale (NOS) for assessing the quality of nonrandomised studies in meta-analyses. Ottawa Hospital Research Institute. The Ottawa Hospital Research Institute. 2014. http://www.ohri.ca/programs/clinical_ epidemiology/oxford.asp. Accessed 1 May 2015.

12. Crombie IK. The pocket guide to critical appraisal : a handbook for health care professionals. London: BMJ; 1996.

13. Bunn F, Goodman C, Pinkney E, Drennan VM. Specialist nursing and community support for the carers of people with dementia living at home: an evidence synthesis. Health Soc Care Community. 2015;24(1):48-67. 
14. Reed MC, Wood V, Harrington R, Paterson J. Developing stroke rehabilitation and community services: a meta-synthesis of qualitative literature. Disabil Rehabil. 2012;34(7):553-63.

15. Schadewaldt V, McInnes E, Hiller JE, Gardner A. Views and experiences of nurse practitioners and medical practitioners with collaborative practice in primary health care-an integrative review. BMC Fam Pract. 2013;14(1):132-43.

16. Pit SW, Hansen V. Factors influencing early retirement intentions in Australian rural general practitioners. Occup Med (Oxf). 2014;64(4):297-304

17. Van Greuningen M, Heiligers PJ, Van der Velden LFJ. Motives for early retirement of self-employed GPs in the Netherlands: a comparison of two time periods. BMC Health Serv Res. 2012;12(1):467-81.

18. Wakeford R, Roden M, Rothman A. General practitioners' retirement plans and what influences them. BMJ. 1986;292(6531):1307-9.

19. Onyura B, Bohnen J, Wasylenki D, Jarvis A, Giblon B, Hyland R, Silver I, Leslie K. Reimagining the self at late-career transitions: how identity threat influences academic physicians' retirement considerations. Acad Med. 2015;90(6):794-801.

20. Vaismoradi M, Turunen $\mathrm{H}$, Bondas T. Content analysis and thematic analysis: implications for conducting a qualitative descriptive study. Nurs Health Sci. 2013;15(3):398-405.

21. Elo $\mathrm{S}$, Kyngäs $\mathrm{H}$. The qualitative content analysis process. J Adv Nurs. 2008;62(1):107-15

22. Batchelor AJ. Senior women physicians: the question of retirement. N Y State J Med. 1990;90(6):292-4.

23. French $F$, Andrew J, Awramenko M, Coutts H, Leighton-Beck L, Mollison J, Needham G, Scott A, Walker K. Why do work patterns differ between men and women GPs? J Health Organ Manag. 2006;20(2-3):163-72.

24. Hill KB, Burke FJT, Brown J, Macdonald EB, Morris AJ, White DA, Murray K. Dental practitioners and ill health retirement: a qualitative investigation into the causes and effects. Br Dent J. 2010;209(5):E8.

25. Jacobson D, Eran M. Expectancy theory components and non-expectancy moderators as predictors of physicians' preference for retirement. J Occup Health Psychol. 1980;53(1):11-26.

26. Newton J, Luce A, van Zwanenberg T, Firth-Cozens J. Job dissatisfaction and early retirement: a qualitative study of general practitioners in the Northern Deanery. Prim Health Care Res Dev. 2004;5(1):68-76.

27. Quandango JS. Career continuity and retirement plans of men and women physicians: the meaning of disorderly careers. Work Occup. 1978;5(1):55-74.

28. Sansom A, Calitri R, Carter M, Campbell J. Understanding quit decisions in primary care: a qualitative study of older GPs. BMJ Open. 2016;6(2):e010592.

29. Silver MP, Pang NC, Williams SA. "Why give up something that works so well?": retirement expectations among academic physicians. Educ Gerontol. 2015;41(5):333-47.

30. Eagles JM, Adie K, Brown T. Retirement intentions of consultant psychiatrists. Psychiatr Bull. 2005;29(10):374-6.

31. Rayburn WF, Strunk AL, Petterson SM. Considerations about retirement from clinical practice by obstetrician-gynecologists. Am J Obstet Gynecol. 2015;213(3):335.e1-4.

32. Sibbald B, Bojke C, Gravelle H. National survey of job satisfaction and retirement intentions among general practitioners in England. BMJ. 2003;326(22):4.

33. Rittenhouse DR, Mertz E, Keane D, Grumbach K. No exit: an evaluation of measures of physician attrition. Health Serv Res. 2004;39(5):1571-88.

34. Orkin FK, McGinnis SL, Forte GJ, Peterson MD, Schubert A, Katz JD, Berry AJ, Cohen NA, Holzman RS, Jackson SH, Martin DE, Garfield JM. United States anesthesiologists over 50: retirement decision making and workforce implications. Anesthesiology. 2012;117(5):953-63.

35. Sutinen R, Kivimäki M, Elovainio M, Forma P. Associations between stress at work and attitudes towards retirement in hospital physicians. Work Stress. 2005;19(2):177-85

36. French FH, Andrew JE, Awramenko M, Coutts H, Leighton-Beck L, Mollison J, Needham G, Scott A, Walker KA. Consultants in NHS Scotland: a survey of work commitments, remuneration, job satisfaction and retirement plans. Scott Med J. 2004:49(2):47-52.

37. Anderson BL, Hale RW, Salsberg E, Schulkin J. Outlook for the future of the obstetrician-gynecologist workforce. Am J Obstet Gynecol. 2008;199(1):88.e1-8.

38. Fletcher $S L$, Schofield DJ. The impact of generational change and retirement on psychiatry to 2025. BMC Health Serv Res. 2007;7(1):141-8.

39. Farley FA, Kramer J, Watkins-Castillo S. Work satisfaction and retirement plans of orthopaedic surgeons 50 years of age and older. Clin Orthop Relat Res. 2008;466(1):231-8
40. Draper B, Winfield S, Luscombe G. The older psychiatrist and retirement. Int J Geriatr Psychiatry. 1997;12(2):233-9.

41. Mears A, Kendall T, Katona C, Pashley C, Pajak S. Retirement intentions of older consultant psychiatrists. Psychiatr Bull. 2004:28(4):130-2.

42. Joyce CM, Wang WC, McDonald HM. Retirement patterns of Australian doctors aged 65 years and older. Aust Health Rev. 2015;39(5):582-7.

43. Evans RW, Ghosh K. A survey of headache medicine specialists on career satisfaction and burnout. Headache. 2015;55(10):1448-57.

44. Heponiemi T, Kouvonen A, Vanska J, Halila H, Sinervo T, Kivimaki M, Elovainio M. Health, psychosocial factors and retirement intentions among Finnish physicians. Occup Med (Oxf). 2008;58(6):406-512.

45. Peisah C, Wijeratne C, Waxman B, Vonau M. Adaptive ageing surgeons. ANZ J Surg. 2014;84(5):311-5.

46. Dodds DW, Cruz OA, Israel H. Attitudes toward retirement of ophthalmology department chairs. Ophthalmology. 2013;120(7):1502-5.

47. Reuben DB, Silliman RG. Lessons from elderly physicians: reflections on practice, changes in medicine, and retirement. J Appl Gerontol. 1988;7(1):49-59

48. Deitch $\mathrm{CH}$, Sunshine JH, Chan WC, Owen JB, Shaffer KA. How Us radiologists use their professional time - factors that affect work activity and retirement plans. Radiology. 1995;194(1):33-40.

49. Landon BE, Reschovsky JD, Pham HH, Blumenthal D. Leaving medicine: the consequences of physician dissatisfaction. Med Care. 2006:44(3):234-42.

50. Grauer H, Campbell N. The aging physician and retirement. Can J Psychiatry. 1983;28(7):552-4

51. Brett TD, Arnold-Reed DE, Hince DA, Wood IK, Moorhead RG. Retirement intentions of general practitioners aged 45-65 years. Med J Aust. 2009;191(2):75-7.

52. Davidson JM, Lambert TW, Parkhouse J, Evans J, Goldacre MJ. Retirement intentions of doctors who qualified in the United Kingdom in 1974: postal questionnaire survey. J Public Health (Oxf). 2001;23(4):323-8.

53. Shanafelt TD, Raymond M, Kosty M, Satele D, Horn L, Pippen J, Chu Q Chew H, Clark WB, Hanley AE, Sloan J, Gradishar WJ. Satisfaction with worklife balance and the career and retirement plans of US oncologists. J Clin Oncol. 2014;32(11):1127-35.

54. Meghea C, Sunshine JH. Retirement patterns and plans of radiologists. AJR Am J Roentgenol. 2006;187(6):1405-11.

55. Davidson JM, Lambert TW, Goldacre MJ, Parkhouse J, MacDonald R. UK senior doctors' career destinations, job satisfaction, and future intentions: questionnaire survey/commentary. Br Med J. 2002;325(7366):685-6.

56. Baker FM. A survey of retirement planning by Texas psychiatrists. J Geriatr Psychiatry Neurol. 1993;6(1):14-9.

57. Goldberg R, Thomas H, Penner L. Issues of concern to emergency physicians in pre-retirement years: a survey. J Emerg Med. 2011:40(6):706-13.

58. Austrom MG, Perkins AJ, Damush TM, Hendrie HC. Predictors of life satisfaction in retired physicians and spouses. Soc Psychiatry Psychiatr Epidemiol. 2003;38(3):134-41.

59. Baker FM. Retirement planning by black psychiatrists. J Geriatr Psychiatry Neurol. 1994;7(3):184-8.

60. Carrière Y, Galarneau D. Delayed retirement: a new trend? Perspectives Labour Income. 2011;23(4):4-16.

61. Grondin SC, Schieman C, Kelly E, Darling G, Maziak D, Mackay MP, Gelfand G. A look at the thoracic surgery workforce in Canada: how demographics and scope of practice may impact future workforce needs. Can J Surg. 2013;56(4):E75-81.

62. McDonald L, Donahue P. Retirement lost? Can J Aging. 2011;30(03):401-22.

63. Gregory ST, Menser T. Burnout among primary care physicians: a test of the areas of work life model. J Healthc Manag. 2015;60(2):133-48.

64. Degen C, Li J, Angerer P. Physicians' intention to leave direct patient care: an integrative review. Hum Resour Health. 2015;13(1):74-86.

65. Győrffy Z, Dweik D, Girasek E. Workload, mental health and burnout indicators among female physicians. Hum Resour Health. 2016;14:12.

66. Kim JE, Moen P. Retirement transitions, gender, and psychological wellbeing: a life-course, ecological model. J Gerontol B Psychol Sci Soc Sci. 2002:57(3):212-P222

67. Noone JH, Stephens C, Alpass FM. Preretirement planning and well-being in later life a prospective study. Res Aging. 2009;31(3):295-317.

68. Wong JY, Earl JK. Towards an integrated model of individual, psychosocial, and organizational predictors of retirement adjustment. J Vocat Behav. 2009;75(1):1-13. 
69. Chambers M, Colthart I, McKinstry B. Scottish general practitioners' willingness to take part in a post-retirement retention scheme: questionnaire survey. BMJ. 2004;328(7435):329.

70. Barrick MR, Mount MK, Li N. The theory of purposeful work behavior: the role of personality, higher-order goals, and job characteristics. Acad Manage Rev. 2013;38(1):132-53.

71. Rosvold E, Bjertness E. Physicians who do not take sick leave: hazardous heroes? Scand J Public Health. 2001;29(1):71-5.

72. Hedden L, Barer ML, Cardiff K, McGrail KM, Law MR, Bourgeault IL. The implications of the feminization of the primary care physician workforce on service supply: a systematic review. Hum Resour Health. 2014;12:32.

73. Roberfroid D, Leonard C, Stordeur S. Physician supply forecast: better than peering in a crystal ball? Hum Resour Health. 2009;7:10

74. Baker FM, Hishinuma ES. AMA and NMA members: retirement planning and definition of a good quality of life. J Geriatr Psychiatry. 2002;35(2):169-92.

75. Bieliauskas LA. Cognitive changes and retirement among senior surgeons (CCRASS): results from the CCRASS Study. J Am Coll Surg. 2008;207(1):69-9.

76. Burke FJ, Main JR, Freeman R. The practice of dentistry: an assessment of reasons for premature retirement. Br Dent J. 1997;182(7):250-4.

77. Davidson JM, Lambert TW, Goldacre MJ. Career pathways and destinations 18 years on among doctors who qualified in the United Kingdom in 1977: postal questionnaire survey. Br Med J. 1998;317(7170):1425-8.

78. De Santo N, Altucci P, Heidland A, Stein G, Cameron J, Rutkowski B. The role of emeriti and retired professors in medicine. QJM. 2014;107(5):405-7.

79. Donner EM, Sze G, Bluth El. Retirement issues for radiologists: consensus statement on successful planning by the commission on human resources of the ACR. J Am Coll Radiol. 2015;12(3):235-8.

80. Draper B, Gething L, Fethney J, Winfield S. The Senior Psychiatrist Survey III: attitudes towards personal ageing, life experiences and psychiatric practice. Aust N Z J Psychiatry. 1999;33(5):717-22.

81. Florence LS, Feng S, Foster CE, Fryer JP, Olthoff KM, Pomfret E, Sheiner PA, Sanfey H, Bumgardner GL. Academic careers and lifestyle characteristics of 171 transplant surgeons in the ASTS. Am J Transplant. 2011;11(2):261-71.

82. Gee WF, Holtgrewe HL, Albertsen PC, Cooper TP, Fenninger RB, Litwin MS, Manyak MJ, Meyer JJ, Miles BJ, O'Leary MP, Painter MR, Rohner TJ, Thomas R, Blizzard RT, Emmons L. Subspecialization, recruitment and retirement trends of American urologists. J Urol. 1998;159(2):509-11.

83. Greenfield L, Proctor MC. Attitudes toward retirement. A survey of the American Surgical Association. Ann Surg. 1994;220(3):382-90.

84. Jonasson O, Kwakwa F. Retirement age and the work force in general surgery. Ann Surg. 1996;224(4):574-82.

85. Kendell RE, Pearce A. Consultant psychiatrists who retired prematurely in 1995 and 1996. Psychiatr Bull. 1997;21:741-5.

86. Lee HJ, Drag LL, Bieliauskas LA, Langenecker SA, Graver C, O'Neill J, Greenfield $L$. Results from the cognitive changes and retirement among senior surgeons self-report survey. J Am Coll Surg. 2009;209(5):668-71.

87. Lee J, Lenzmeier T, Boulger J, Buck S, Bergeron D, Hill TJ. Retirement of senior physicians in rural Minnesota. Factors influencing physicians' plans to retire. Minn Med. 1995;78(12):21.

88. Moriarity AK, Brown ML, Schultz LR. Work and retirement preferences of practicing radiologists as a predictor of workforce needs. Acad Radiol. 2014;21(8):1067-71.

89. McGuirt WF, McGuirt WF. Otolaryngology retirement profile in the southeastern United States. Laryngoscope. 2002;112(2):213-5.

90. Rowe ML. Health, income, and activities of retired physicians. N Y State Med. 1989;89(8):450-3.

91. Smith KA, Sommer DD, Grondin S, Rotenberg B, Tewfik MA, Kilty S, Wright E, Janjua A, Lee J, Diamond C, Rudmik L. Assessment of the current Canadian rhinology workforce. J Otolaryngol Head Neck Surg. 2015;44(1):15.

\section{Submit your next manuscript to BioMed Central and we will help you at every step:}

- We accept pre-submission inquiries

- Our selector tool helps you to find the most relevant journal

- We provide round the clock customer support

- Convenient online submission

- Thorough peer review

- Inclusion in PubMed and all major indexing services

- Maximum visibility for your research

Submit your manuscript at www.biomedcentral.com/submit
Biomed Central 